Archived version from NCDOCKS Institutional Repository http://libres.uncg.edu/ir/asu/

\title{
Appalacȟnan
}

B O O NE, NORTH CAROLINA

\section{VA Community Mental Health Service Providers' Utilization of and Attitudes Toward Telemental Health Care: The Gatekeeper's Perspective}

By: John Paul Jameson, Mary Sue Farmer, Katharine J. Head, John Fortney \& Cayla R. Teal

\begin{abstract}
Context: Mental health $(\mathrm{MH})$ providers in community-based outpatient clinics (CBOCs) are important stakeholders in the development of the Veterans Health Administration (VA) telemental health (TMH) system, but their perceptions of these technologies have not been systematically examined. Purpose: The purpose of this study was to investigate the attitudes of CBOC providers about TMH services, current utilization of these technologies in their clinics, and sources of knowledge regarding TMH.

Method: The study employed a mixed-methods design to examine aspects of TMH in CBOCs located in a VA network in the south-central United States. Semi-structured, on-site group interviews conducted with $86 \mathrm{CBOC} \mathrm{MH}$ providers were followed by in-depth phone surveys with an $\mathrm{MH}$ provider identified as a key informant at each of 36 CBOCs in the VA network.

Findings: The utilization of TMH services varied widely between CBOCs, and the scope of services provided typically focused on delivery of medication management, with little provision of psychological services. Further, several important barriers to expanded use of TMH were identified, including limited education and training and shortage of dedicated space for TMH encounters. Conclusions: General attitudes toward TMH were positive, and most CBOC providers indicated that they would like to expand use of TMH in their clinics.
\end{abstract}

John Paul Jameson, Mary Sue Farmer, Katharine J. Head, John Fortney \& Cayla R. Teal (2011) "VA Community Mental Health Service Providers' Utilization of and Attitudes Toward Telemental Health Care: The Gatekeeper's Perspective"Journal of Rural Health \#24 pp.425-432 Version of Record Available From (onlinelibrary.wiley.com) 


\title{
VA Community Mental Health Service Providers' Utilization of and Attitudes Toward Telemental Health Care: The Gatekeeper's Perspective
}

\begin{abstract}
A large number of veterans reside in rural areas, ${ }^{1}$ and providing mental health $(\mathrm{MH})$ services for this widely dispersed population presents an immense challenge. In the past 2 decades, the Department of Veterans Affairs (VA) has constructed a system of satellite clinics, known as community-based outpatient clinics (CBOCs), to increase accessibility of primary $\mathrm{MH}$ services for rural veterans. ${ }^{2}$ Concurrently, the VA has invested heavily in telemental health (TMH) technologies to provide a wide array of psychological and psychiatric services to address the needs of rural veterans. ${ }^{3,4}$ TMH services, usually originating at a VA medical center, are commonly delivered to veterans in CBOCs through videoconferencing equipment. During fiscal year 2008, the VA provided care to 230,000 patients via interactive video and provided $\mathrm{TMH}$ to veterans in 300 CBOCs. ${ }^{5}$ The number of TMH encounters, number
\end{abstract}


of veterans served, and number of CBOCs utilizing TMH services are all expected to grow in the coming years.

Though initial data suggest the potential value of TMH for the widespread provision of $\mathrm{MH}$ services to veterans, the number of studies is limited, and these studies have not focused on the perspectives of CBOC providers. ${ }^{6}$ The few existing studies of TMH in VA community settings have suggested that TMH can be an effective medium for the delivery of both $\mathrm{MH}$ assessments ${ }^{7,8}$ and interventions. $^{9-12}$ The VA is continuing to develop a research base to demonstrate the effectiveness of TMH in veteran populations. ${ }^{13}$

Investigations in non-VA settings suggest that TMH appears to be acceptable to consumers and providers. ${ }^{14}$ Numerous studies of consumers have suggested that TMH would be an acceptable alternative to face-to-face care. ${ }^{7,15-17}$ Rural consumers appear to be particularly receptive to TMH services. ${ }^{16}$ In general, providers also seem to recognize the value of TMH services and endorse a willingness to use such services if they improve access to care for consumers. ${ }^{17}$ Rural primary care providers using TMH consultations have reported a particularly high level of satisfaction with the quality of services. ${ }^{16,18}$

Though studies in non-VA settings may serve as a guide, the generalizability of such research to CBOC staff is questionable for several reasons. First, research on non-VA providers' acceptance of TMH use has often addressed specific applications of TMH (eg, provision of psychotherapy for depression or medication consultations). Broader clinical applications of TMH may result in different provider attitudes. Other assessments ${ }^{17}$ have examined providers and patients not currently using TMH and instead gathered acceptability judgments based upon hypothetical scenarios (ie, whether use of TMH would be acceptable if the technology and infrastructure were available). These opinions may not accurately reflect the strengths and weaknesses of TMH as it is currently applied in clinical settings.

Second, providers in non-VA settings may be less likely to be mandated to incorporate TMH into community practice. VA guidelines require that a broad range of $\mathrm{MH}$ services be provided within CBOCs, and TMH is often the only option available to deliver these required services. ${ }^{3}$ Moreover, TMH equipment has been provided to most CBOCs by the VA, with the expectation that this equipment will be used to deliver patient care. Thus, the decision to adopt TMH for clinical practice may be considered an authority innovation-decision, a decision made by administrative personnel and not by staff who would be using the innovation in practice. ${ }^{19}$ The adoption of innovations by authority innovation-decisions does not necessarily lead to true adoption by the users of an innovation but may instead lead to superficial adoption to meet the minimum requirements of the administrative system.

To maximize the return on the investment in TMH by the VA, a clear understanding of attitudes and barriers faced by CBOC providers is critical. Though CBOC providers do not necessarily provide TMH services themselves, they may often refer their patients for TMH to specialty service providers as a means to reduce case overload and, therefore, act as important gatekeepers to accessing TMH services.

The present study attempts to address some of these gaps in our current knowledge. Data were collected from $\mathrm{MH}$ providers in CBOCs within a large, predominantly rural area of the country using a mixed-methods design. The purpose of the study was 3-fold. First, we wished to examine current utilization of TMH services by CBOCs and assess the range of services provided. Second, we wished to assess providers' comfort with using the technologies, as well as determine the providers' training in TMH services. Third, we wished to examine providers' attitudes and perceived barriers to the use of TMH for the delivery of various services. The study was not designed to test a priori hypotheses regarding utilization or attitudes but to provide a description of services as perceived by providers in CBOCs.

\section{Method \\ Participants}

The study was conducted with CBOC MH staff located in Veterans Integrated Service Network (VISN) 16. VISN 16 serves approximately 445,000 veterans in 8 states in the southeastern and south-central United States. ${ }^{20}$ The service area features the highest number of veterans of the conflicts in Iraq and Afghanistan who utilize the VA health care system. ${ }^{21}$ Almost half of veterans in VISN 16 reside in rural areas. ${ }^{22}$ Many of these veterans are served by the 40 CBOCs located in the network at the time of this study; according to rural-urban continuum codes, ${ }^{23}$ 29 of these 40 CBOCs are located in nonmetropolitan areas.

The study consisted of 2 parts: semistructured, on-site group interviews conducted with all willing and available CBOC MH staff, followed by in-depth phone surveys with an MH provider identified by lead MH administrators at the parent VA facility (ie, the medical center with administrative responsibilities for the CBOC) as a key informant at each CBOC. Phone surveys were conducted 3-6 months after the on-site group interviews. We note that there was participant overlap between the interviews and the survey; most participants in the phone surveys had also participated in the group interviews. All data were collected in 2008 and 2009. 


\section{Procedure}

All study procedures were approved by the Baylor College of Medicine Institutional Review Board. The study followed a sequential mixed-methods design, with the semistructured group interviews preceding the in-depth phone surveys. A mixed-methods design was employed to gain a clear picture of pragmatic issues that exist with TMH as it is used in CBOCs. As discussed above, it is unclear whether extant literature captures the experiences and attitudes of $\mathrm{CBOC}$ providers. Therefore, a strictly quantitative design would run the risk of omitting concerns salient to providers. A qualitative component was necessary to ensure that providers would be able to voice concerns in a relatively open forum.

\section{Site-based Mental Health Service Provider Group Interviews}

The semistructured interviews were conducted by pairs of researchers from the South Central Mental Illness Research, Education and Clinical Center (MIRECC). The pairs of researchers conducted 1 group-based MH staff interview at each visited CBOC. Sites were contacted and informed of the study's purpose via letter from the VISN 16 network director several weeks prior to the visit. A 1- to 2-hour group meeting (with as many MH service providers as could attend) was scheduled, after which the 2 researchers met individually with selected providers. СВOC providers were assured of confidentiality in all discussions. CBOC providers were asked a series of questions that generally included provider description of the CBOC's veteran population, clinic organization, education and training needs, clinic and community resources, and interest in participating in research. The researchers independently collected detailed field notes regarding staff's answers to these questions. It is important to note that specific questions regarding TMH were not included in the semistructured interviews. Mentions of TMH emerged in the context of these general areas of inquiry.

At the end of each site visit, each pair of researchers completed a standardized site-visit report of its observations about the clinic and staff by reviewing and discussing their independent field notes from the interviews. A content analysis of these reports was conducted by the Houston-based team members (Jameson and Teal). An iterative review process of the reports was utilized to develop categories within the areas described above, as well as relationships across these areas. The reviewers conducted independent reviews of each report, carrying out direct coding of report substance, and then met to collectively review the coded re- ports and identify thematic content. Reviewers developed and decided thematic categories, references between categories, and positive and negative examples within the categories. Incongruities of interpretations were resolved through extensive discussion and continued review of additional exemplars until consensus in interpretation was achieved. Other researchers who conducted the site visits, as well as participants at selected CBOCs, were asked to review the preliminary results and offer comments regarding accuracy of the analysis, needed clarifications, and other comments. The final analysis incorporated these validation activities.

\section{Telephone Survey}

TMH questions were embedded into a larger telephone survey investigating various aspects of clinical practice and educational needs. A survey instrument, adapted from an existing telemedicine survey, ${ }^{24}$ was constructed with 3 goals in mind: (1) to corroborate qualitative findings from the site visits, (2) to measure attitudes regarding specific uses of TMH, and (3) to gather more detailed information on current utilization and practices. To accomplish these goals, questions from the existing TMH survey were reworded to specifically address the context of MH care. Further, items irrelevant to the VA setting (eg, Medicare reimbursement, financial investment in technology) were omitted. Items pertinent to TMH are listed in Tables 1 and 2.

Respondents rated agreement with 10 statements reflecting positive and negative attitudes toward factors in use of TMH that emerged in the staff interviews,

Table 1 Reported Use and Effectiveness Ratings of Services Delivered via Telemental Health

\% Respondents \% Referring/ Mean Effectiveness Indicating Use \% Providing* Ratings (SD)**

\begin{tabular}{lccc}
\hline Any use & 87.9 & - & - \\
Administrative use & 54.5 & - & - \\
Continuing education & 21.2 & - & - \\
Any clinical use & 57.6 & $84.2 / 15.8$ & - \\
Medication & & & $1.64(0.65)$ \\
Management & 36.4 & $92.3 / 7.8$ & $0.79(1.34)$ \\
Diagnostic interviews & 12.1 & $100 / 0$ & $0.27(1.44)$ \\
Psychotherapy & 9.1 & $33.3 / 66.7$ & $1.42(0.66)$ \\
Second opinions & 6.1 & $100 / 0$ & \\
N $=33$. & \\
*Indicates the proportion of respondents & referring veterans in the \\
community-based outpatient clinic for a service via telemental health ver- \\
sus the proportion of respondents providing a service via telemental health \\
to veterans in other locations. \\
**Respondents rated effectiveness on a 4-point scale $(-2$ to +2$)$; higher \\
scores indicate higher effectiveness ratings.
\end{tabular}


Table 2 Agreement With Positive and Negative Statements Regarding Telemental Health Utilization.

\begin{tabular}{|c|c|c|c|}
\hline & $\mathrm{N}^{*}$ & Mean $(S D)^{* *}$ & $\begin{array}{l}\text { \%Respondents } \\
\text { Indicating } \\
\text { Agreement*** }\end{array}$ \\
\hline $\begin{array}{l}\text { The quality of TMH sound } \\
\text { and video is adequate for } \\
\text { clinical use }\end{array}$ & 31 & $0.84(1.27)$ & 80.7 \\
\hline $\begin{array}{l}\text { More research is needed on } \\
\text { the effectiveness of TMH }\end{array}$ & 33 & $0.94(1.20)$ & 78.8 \\
\hline $\begin{array}{l}\text { I do not like the loss of } \\
\text { contact associated with } \\
\text { TMH }\end{array}$ & 33 & $0.91(1.18)$ & 75.8 \\
\hline $\begin{array}{l}\text { CBOCs could address } \\
\text { clinical overload with } \\
\text { more TMH use }\end{array}$ & 33 & $0.67(1.41)$ & 69.7 \\
\hline $\begin{array}{l}\text { MH services can easily be } \\
\text { adapted to use of } \\
\text { interactive video }\end{array}$ & 33 & 0.45 (1.39) & 66.6 \\
\hline $\begin{array}{l}\text { Access to TMH is limited by } \\
\text { other uses of the room } \\
\text { and equipment }\end{array}$ & 33 & $0.42(1.62)$ & 63.7 \\
\hline $\begin{array}{l}\text { There is adequate tech } \\
\text { support available for TMH } \\
\text { use }\end{array}$ & 31 & $0.03(1.49)$ & 54.8 \\
\hline $\begin{array}{l}\text { I have been adequately } \\
\text { trained to use TMH }\end{array}$ & 33 & $0.00(1.56)$ & 54.6 \\
\hline $\begin{array}{l}\text { Technical problems } \\
\text { interfere with } \mathrm{TMH} \\
\text { sessions }\end{array}$ & 30 & $-0.03(1.40)$ & 50.0 \\
\hline $\begin{array}{l}\text { I do not know enough about } \\
\text { TMH to use it to expand } \\
\text { my caseload }\end{array}$ & 33 & $-0.88(1.52)$ & 27.3 \\
\hline
\end{tabular}

TMH, telemental health; COBCs, community-based outpatient clinics; $\mathrm{MH}$, mental health.

${ }^{*} \mathrm{~N}$ varies due to nonresponse to questions.

**Range -2 to +2 ; higher values indicate stronger agreement.

***Respondents indicating that they strongly agree or somewhat agree.

using a 4 -point Likert scale $(-2=$ strongly disagree, $-1=$ somewhat disagree, $1=$ somewhat agree, 2 = strongly agree). These statements reflected satisfaction with the technology and the technology support received, acceptability of TMH compared with face-to-face contact, satisfaction with the current body of research on $\mathrm{TMH}^{\prime} \mathrm{s}$ clinical effectiveness, satisfaction with training and knowledge regarding $\mathrm{TMH}$, ability of $\mathrm{TMH}$ to reduce caseloads, adaptability of services to a TMH medium, and limitations of TMH availability due to space or use of equipment for other purposes.

To examine perceived effectiveness of services delivered via TMH, respondents rated their agreement with statements describing the clinical effectiveness of specific services delivered via telehealth (eg, "Telemental health is effective for psychological evaluations") on a 4-point
Likert scale (described above). To assess current practices, respondents were asked to estimate the number of times they used TMH for any purpose over the past month and indicate their 2 most common uses of TMH. They were also asked to indicate sources of knowledge and training in $\mathrm{TMH}$ and rate how comfortable they were using TMH for any purpose on a 4-point Likert scale $(-2=$ not at all comfortable, $-1=$ somewhat uncomfortable, $1=$ somewhat comfortable, 2 = very comfortable).

\section{Results}

Interviews were conducted at 36 of the $40(90 \%)$ CBOCs in the VISN. ${ }^{\text {a }}$ Three CBOCs were not visited because no $\mathrm{MH}$ providers were located on-site during the study period. Additionally, I CBOC was in the process of reopening after a natural disaster. A total of $86 \mathrm{MH}$ staff participated in the face-to-face interviews, with a range of 1-7 providers participating in each interview $(M=2.2$, median $=2, \mathrm{SD}=1.96$ ). This represented $79 \%$ of all $\mathrm{MH}$ providers in these CBOCs at the time of this study and included social workers, psychologists, psychiatrists, addiction therapists, and, to a lesser extent, nurses and physician assistants.

Thirty-three of the 36 eligible providers responded to the survey (a response rate of 91.7\%): 49\% $(\mathrm{n}=16)$ social workers; $21.2 \%(\mathrm{n}=7)$ doctoral-level psychologists; $21.2 \%(\mathrm{n}=7)$ psychiatrists; $6 \%(\mathrm{n}=2)$ nurse practitioners; and 3\% (n=1) physician assistants. Duration of years employed at the CBOC was as follows: $21 \%(\mathrm{n}=$ 7) employed less than 1 year, $33.3 \%(n=11)$ employed $1-3$ years, $21 \%(n=7)$ employed $4-5$ years, and $24 \%$ $(\mathrm{n}=8)$ employed 5 or more years. The relatively large number of new employees was not surprising, given that many CBOCs in the VISN have opened within the last several years and spending on $\mathrm{MH}$ staff has grown considerably.

\section{Provider Interviews}

Content analysis revealed that 21 of the 36 site-visit reports $(58 \%)$ contained specific mention of TMH. Most comments in the site-visit reports were relatively brief and written in a shorthand style, though 3 of the reports featured comments on TMH that exceeded 2 paragraphs. Three salient themes emerged within the text of many of the site-visit reports.

\section{Current Utilization}

Ten of the 21 site-visit reports contained descriptions of current TMH utilization. Reports mentioned both clinical and administrative applications of the TMH system. The 
most commonly mentioned clinical service provided via TMH was pharmacotherapy (mentioned in 8 of the 10 reports discussing TMH services), with psychological services mentioned in only 2 reports.

The extent to which TMH was used appeared to vary widely between CBOCs. Site-visit reports indicated that CBOCs use TMH to supplement the services available on-site (eg, "Psychiatrist [provides services] as needed"), or to occasionally cover services generally available within the CBOC (eg, "Not using [telemental health equipment] very much, though [a psychiatrist] has done some 'substitute' pharmacotherapy on a very infrequent basis"). Other CBOCs appeared to rely heavily on TMH for the provision of $\mathrm{MH}$ services, especially pharmacotherapy, as noted in this report from a smaller, more remote CBOC: "Currently provider services are seldom provided in-house, with only 1 full day (split into 2 halfdays) of VAMC-offered telemental health and little primary care assistance."

Still other reports suggested very little utilization of $\mathrm{TMH}$. One report attributed this to a lack of understanding and education ("There is virtually no understanding of telemental health for any purpose-of the procedure, the process, the utility, etc.").

\section{Clinical Office Space Often a Barrier to Telemental Health Provision}

Eight reports featured comments on the space available within the CBOC for TMH service use, with 6 of these noting inadequate accommodations for TMH equipment (eg, "Space restrictions limit use of telemedicine."). Reports suggested that 2 sites had adequate space, with 1 report noting that space for $\mathrm{TMH}$ is a point of envy from providers at other CBOCs: "This CBOC has a huge telemental health/group room. [Staff from other CBOCs] commented how much they wished they had a space like this CBOC."

\section{Providers' Wish to Expand Telemental Health}

Eleven site-visit reports mentioned CBOC providers' desire to expand the use of TMH. Specifically, 7 reports note that providers would like to use the TMH system to expand the availability and spectrum of clinical services. Four of these 7 reports expressed a desire to expand utilization in direct response to the limited MH staff available on-site. Other reports indicated that expansion was desired to increase continuing education opportunities ( 2 reports) or promote collaborations ( 2 reports) with MIRECC researchers.

\section{Telephone Survey}

To account for $\mathrm{MH}$ resources available on-site, phonesurvey data were stratified by the presence of prescribing $\mathrm{MH}$ providers (psychiatrists or dedicated nurse practitioners or physician assistants) at the CBOC of each respondent and compared. No substantial differences in reported utilization of TMH referrals were found between respondents with a prescribing provider on-site and those with no prescribing provider on-site. Therefore, the current sample was analyzed as a single group.

Most of the 33 phone-survey respondents ( $\mathrm{n}=29$; $87.9 \%$ ) indicated that they have used TMH for administrative or clinical purposes. The number of uses of TMH reported by respondents over the month prior to the survey varied widely (range $=0-103$ unique uses, median $=3$ ). As noted in Table 1 , the most commonly reported clinical use of TMH was for medication management; use for provision of psychological services (diagnostic interviews, psychological assessments, and psychotherapy) was less commonly reported. Further, respondents reported referring veterans for TMH services more frequently than providing TMH services to veterans at other CBOCs.

Mean ratings of the effectiveness of services delivered via TMH are also presented in Table 1. Each of the 4 services was rated positively. With the exception of second opinions, effectiveness ratings for services closely mirror reported use of the services.

Few respondents $(\mathrm{n}=5,15.2 \%)$ identified formal training programs as an important source of knowledge of TMH. The most frequently identified source of TMH knowledge was personal experience ( $\mathrm{n}=24,72.7 \%$ ). Other identified sources of knowledge included information from colleagues $(\mathrm{n}=14,42.4 \%)$, professional association meetings $(\mathrm{n}=7,21.2 \%)$, presentations given in the VA $(n=6,18.2 \%)$, medical literature $(n=5,15.2 \%)$, medical or graduate training $(\mathrm{n}=3,9.1 \%)$, electronic media $(\mathrm{n}=3,9.1 \%)$, and the mass media $(\mathrm{n}=1,3.0 \%)$.

Respondents' ratings of agreement with positive and negative aspects of TMH use are presented in Table 2 . Providers endorsed both positive aspects and negative aspects of current TMH practice. Most participants indicated that the transmission quality of the TMH equipment was sufficient, and that TMH had the potential to reduce case overload in CBOCs. Most participants also indicated that they believed more research on the effectiveness of interventions delivered via TMH is needed, and that the loss of face-to-face contact with patients is not desirable. Respondents' opinions were generally split on seeing technical problems as frequently problematic and believing they were adequately trained to use $\mathrm{TMH}$ appropriately. 


\section{Discussion}

Using a mixed-methods design, the present study examined attitudes of CBOC MH clinicians toward use of TMH services, as well as current utilization of these technologies. The study represents an essential first step in understanding the complex benefits and problems in the widespread application of TMH in routine VA care and highlights the importance of $\mathrm{CBOC} \mathrm{MH}$ providers as key stakeholders in improving and expanding the $\mathrm{TMH}$ system in the VA. The data also suggest that improving the TMH system in the VA is likely to require a multifaceted approach that considers the important role of CBOC MH providers.

Both the site-visit reports and phone surveys of key informants indicate that utilization of TMH technologies for the provision of clinical care varies widely between CBOCs. Though a slight majority of survey respondents reported regular use of TMH for referrals for clinical services, a substantial proportion of respondents did not. The survey results corroborated the analysis of the site-visit reports, which varied widely in descriptions of the frequency and purpose of TMH utilization. Some CBOC staff described TMH use as a stopgap measure for temporary staffing issues; others reported it as the primary source of $\mathrm{MH}$ services. However, more research is needed to determine the extent to which low utilization reflects a lack of need for additional services in CBOCs and the extent to which low utilization reported by many participants in this study reflects systemic barriers.

Additionally, the current study suggests that the scope of services offered via TMH is somewhat limited. Medication management accounts for most reported clinical uses; referral for psychological services such as diagnostic interviewing and psychotherapy were reported by few providers. The survey results echo the predominance of pharmacotherapy and relative rarity of psychological services found in the analysis of the site-visit reports. This finding is consistent with other data demonstrating disparities in psychotherapy utilization between rural and urban veterans enrolled in VA care. ${ }^{25}$ It is notable that providers appear to have some reservations about the effectiveness of psychological services delivered via TMH as well, despite promising research evidence to the contrary (for a review, see Bee et al. ${ }^{26}$ ). Publicizing such research findings among $\mathrm{CBOC}$ providers may help to ameliorate some of these concerns. Additionally, the perceptions of the effectiveness of psychotherapy via TMH may reflect a lack of experience among many providers. Past research has demonstrated that referring physicians' satisfaction with TMH services can improve over time with use. ${ }^{18}$ The creation of more training and educational opportunities for $\mathrm{CBOC}$ providers to gain experience with psy- chological services via TMH may improve attitudes and utilization.

Despite the limits of current utilization, СBOC providers appeared optimistic about the utility of $\mathrm{TMH}$; data from both the survey and the site-visit reports suggest that these providers desire expansion of TMH services in their clinics. Expanded use of TMH to address clinician shortages within their СBOC was a consistent theme in the site-visit reports. Further, several interviewees saw the potential for increased access to research and educational opportunities.

Results from the current study highlight some perceived strengths of the VA TMH system. All participants in the study had access to videoconferencing equipment for use in TMH delivery, and most participants reported that the audio and video quality was sufficient for clinical services. This indicates that the VA system has largely resolved many technical problems that can plague TMH systems, especially in rural areas. ${ }^{27}$ This is no small achievement, considering the remoteness of several CBOCs in the study.

However, the results also reveal that the barriers to wider TMH utilization cited by the providers reflect complex systemic issues. First, having adequate space for TMH services revealed itself consistently as a serious challenge. The creation of space for teleconferencing equipment may seem a simple fix. However, because privacy concerns necessitate that the equipment be located in clinical space, it may require the reorganization of exam room space and patient scheduling in these busy primary care settings. Further, $65 \%$ of CBOC space is leased from private owners, and an additional $26 \%$ of CBOCs are private clinics that are contracted with the VA to provide services to veterans. ${ }^{28}$ Therefore, adding space to these structures for TMH may not be feasible in many cases. Placing equipment in a location within the community may be one solution to this problem.

Second, a significant proportion of survey respondents indicated that they did not feel adequately trained to use $\mathrm{TMH}$, and this finding was also evident in the site-visit reports. To maximize the potential of TMH, an understanding of clinical applications is necessary but not sufficient. Knowledge of the technical aspects of the equipment (eg, connecting to other sites, optimizing audio and video quality) and clinical policies and procedures (eg, initiating a referral for TMH services, coordinating between the CBOC and VA medical center for service delivery, establishing and implementing safety measures for homicidal or suicidal veterans using the system) are also necessary for successful and sustainable use of TMH. Working with СBOC providers to identify their specific educational and training needs is critical to designing successful programs to improve providers' comfort with TMH. 
The results of the current study are strengthened by a high response rate and the use of a mixed-method design. Much of the data collected in the phone survey corroborate the thematic analysis of the site-visit reports. Moreover, salient themes regarding TMH services emerged, despite the fact that TMH was not specifically asked about in the interviews.

Despite these strengths, the present study is subject to several important limitations. First, the study was conducted exclusively within the VA system, and the results may not generalize to non-VA settings. Factors such as third-party reimbursement and coordination of medical records for Health insurance Portability and Accountability Act compliance could significantly influence attitudes and utilization in other settings. However, these factors were not examined in the current study, as they have minimal impact on VA TMH services. Second, all participants in both the qualitative portion of the study and the survey were practitioners in VISN 16. Though the VA telehealth program is coordinated at the national level by the Office of Care Coordination, it is not known whether the strengths and weaknesses of the TMH system identified by these providers are unique to this VISN. Therefore, caution is advised regarding generalization of the results to $\mathrm{TMH}$ systems in other areas of the country. Third, because the study was exploratory in nature, direct hypothesis testing was not conducted. Moreover, the study was insufficiently powered to examine attitudinal and utilization differences based on structural variables (eg, rural vs urban location, staff size). Future studies may wish to examine structural factors in order to customize initiatives intended to improve TMH utilization. The data from the current study may serve as an initial step for such work.

The generally positive attitudes among providers in the current study suggest a reason for optimism for the continued development of TMH in the VA health care system. Acceptance of TMH services by these providers is critical to the success of the VA's rural-services strategy, as they represent an important link between specialty providers in VA tertiary care settings and the veterans who rely on CBOCs for their care and commonly provide referrals for TMH care originating at another site. Further, these providers possess unique insight regarding the barriers encountered in the day-to-day use of TMH and can offer meaningful input for the improvement of the care-delivery system.

\section{Endnote}

${ }^{a}$ We note that one of the key informants who completed the phone interview supplied mental health services via telemental health to the 3 CBOCs without mental health providers on-site. Therefore, this participant's responses likely reflect experiences at these 3 CBOCs as well as the participant's home site.

\section{References}

1. United States Department of Veterans Affairs, Veterans Health Administration (2010). Rural Health. Updated March 31, 2010. Retrieved from http://www.ruralhealth. vagov/RURALHEALTH/index.asp on July 12, 2010.

2. Chapko MK, Borowsky SJ, Fortney JC, et al. Evaluation of the Department of Veterans Affairs community-based outpatient clinics. Med Care 2002;40(7):555-560.

3. United States Department of Veterans Affairs, Veterans Health Administration. Uniform Mental Health Services in the VA Medical Centers and Clinic-VHA Handbook 1160.01; Washington, DC: Dept. of Veterans Affairs; 2008.

4. United States Department of Veterans Affairs, Veterans Health Administration. (2009). Care Coordination Services: About us. Retrieved October 8, 2009, from http://www.carecoordination.va.gov/about-us/index.asp.

5. Tuerk PW, Fortney J, Bosworth HB, et al. Toward the development of national telehealth services: the role of veterans health administration and future directions for research. Telemed J E Health 2010;16(1):115-117.

6. Weeks WB, Wallace AE, West AN, Heady HR, Hawthorne $\mathrm{K}$. Research on rural veterans: an analysis of the literature. J Rural Health 2008;24(4):337-344.

7. Dobscha SK, Corson K, Solodsky J. Use if videoconferencing for depression research: enrollment, retention, and patient satisfaction. Telemed J E Health 2005; $11(1): 84-89$.

8. Shore JH, Savin D, Orton H, Beals J, Manson SM. Diagnostic reliability of telepsychiatry in American Indian veterans. Am J Psychiatry 2007;164(1):115-118.

9. Fortney JC, Pyne JM, Edlund MJ, et al. A randomized trial of telemedicine-based collaborative care for depression. J Gen Intern Med 2007;22(8):1086-1093.

10. Frueh BC, Monnier J, Yim E, Grubaugh AL, Hamner MB, Knapp RA. A randomized clinical trial of telepsychiatry for post-traumatic stress disorder. $J$ Telemed Telecare 2007;13(3):142-147.

11. Morland LA, Pierce K, Wong MY. Telemedicine and coping skills groups for Pacific Island veterans with post-traumatic stress disorder: a pilot study. J Telemed Telecare 2005; 10(5):286-289.

12. Ruskin PE, Silver-Aylaian M, Kling MA, et al. Treatment outcomes in depression: comparison of remote treatment through telepsychiatry to in-person treatment. Am J Psychiatr. 2004;161(8):1471-1477.

13. Morland LA, Greene CJ, Rosen C, Mauldin PD, Frueh BC. Issues in the design of a randomized noninferiority clinical trial of telemental health psychotherapy for rural combat veterans with PTSD. Contemp Clin Trials 2009;30(6):513-22. 
14. Richardson LK, Freuh CB, Grubaugh AL, Egede L, Elhai JD. Current directions in videoconferencing tele-mental health research. Clin Psychol (New York). 2009;16(3):323-338.

15. Grubaugh AL, Cain GD, Elhai JD, Patrick SL, Freuh C. Attitudes toward medical and mental health care delivered via telehealth applications among rural and urban primary care patients. J Nerv Ment Dis 2008; 196(2):166-170.

16. Hilty DM, Nesbitt TS, Kuenneth CA, Cruz GM, Hales RE. Rural versus suburban primary care needs, utilization, and satisfaction with telepsychiatric consultation. J Rural Health 2007; 23(2):163-165.

17. Swinton JJ, Robinson WD, Bischoff RJ. Telehealth and rural depression: physician and patient perspectives. Fam Syst Health 2009;27(2):172-182.

18. Hilty DM, Yellowlees DM, Nesbitt TS. Evolution of telepsychiatry to rural sites: changes over time in types of referral and in primary care providers' knowledge, skills and satisfaction. Gen Hosp Psychiatry 2006;28(5):367-373.

19. Rogers E. Diffusion of Innovations. New York, NY: The Free Press; 1995.

20. United States Department of Veterans Affairs, Veterans Health Administration. About VISN 16. 2009. Retrieved October 8, 2009, from http://www.visn 16.va.gov/about/ index.asp.

21. VHA Office of Public Health and Environmental Hazards. Analysis of VA Health Care Utilization Among Operation Enduring Freedom (OEF) and Operation Iraqi Freedom (OIF) Veterans. Washington, DC: US Department of Veterans Affairs; 2010. Retrieved April 8, 2010, from: www.acatoday.org/ppt/4thQtrFY09OEF_OIF_HCU.ppt.
22. South Central Mental Illness Research and Clinical Center. South Central MIRECC Information Sheet; 2009. Retrieved April 20, 2010, from http://www.mirecc.va. gov/docs/infoSheets/VISN_16_Info_Sheet2009.pdf.

23. United States Department of Agriculture Economic Research Service. Measuring rurality: rural-urban continuum codes; 2003. Retrieved November 11, 2004, from http://www.ers.usda.gov/briefing/rurality/ RuralUrbCon/.

24. Barton PL, Brega AG, Devore PA, et al. Specialist physicians' knowledge and beliefs about telemedicine: a comparison of users and nonusers of the technology. Telemed J E Health. 2007;13(5): 39-43.

25. Cully JA, Jameson JP, Phillips LA, Kunik ME, Fortney JC. Use of psychotherapy by rural and urban veterans. J Rural Health. 2010;26:225-233.

26. Bee PE, Bower P, Lovell K, et al. Psychotherapy mediated by remote communication technologies: a meta-analytic review. BMC Psychiatry 2008;8(60). Retrieved April 20, 2010, from: http://www.biomedcentral.com/content/pdf/ 1471-244X-8-60.pdf.

27. Stamm HB. Bridging the rural-urban divide with telehealth and telemedicine. In Stamm HB (ed.). Rural Behavioral Healthcare: An Interdisciplinary Guide. Washington, DC: American Psychological Association; 2003.

28. Panangala SV, Mendez BHP. Veterans Health Administration: Community-Based Outpatient Clinic. Washington, DC: Congressional Research Service; 2010. Retrieved April 8, 2010, from http://assets.opencrs.com/ rpts/R41044_20100128.pdf. 